\title{
Stathmin I increases radioresistance by enhancing autophagy in non-small-cell lung cancer cells
}

\author{
This article was published in the following Dove Press journal: \\ OncoTargets and Therapy \\ 29 April 2016 \\ Number of times this article has been viewed
}

\author{
Xi Zhang ${ }^{1,2}$ \\ Jingfen $\mathrm{ji}^{3}$ \\ Yu Yang ${ }^{4}$ \\ Juan Zhang ${ }^{2}$ \\ Liangfang Shen' \\ 'Department of Oncology, Xiangya \\ Hospital of Central South University, \\ ${ }^{2}$ Department of Oncology, The Third \\ Xiangya Hospital of Central South \\ University, ${ }^{3}$ Department of General \\ Surgery, The Second Xiangya Hospital \\ of Central South University, \\ ${ }^{4}$ Department of Oncology, \\ 163 Hospital of PLA, Changsha, \\ Hunan, People's Republic of China
}

\begin{abstract}
Radioresistance has been demonstrated to be involved in the poor prognosis of patients with non-small-cell lung cancer (NSCLC). However, the underlying mechanism remains largely unclear. Investigation on special therapeutic targets associated with radioresistance shows promises for the enhancement of clinical radiotherapy effect toward NSCLC. This study aimed to reveal the role of Stathmin1 (STMN1) in radioresistance in NSCLC as well as the underlying mechanism. Our data showed that the protein levels of STMN1 were significantly upregulated in NSCLC cells subjected to radiation, accompanied with the activation of autophagy. Knockdown of STMN1 expression enhanced the sensitivity of NSCLC cells to X-ray, and the radiation-induced autophagy was also inhibited. Molecular mechanism investigation showed that knockdown of STMN1 expression upregulated the activity of phosphoinositide 3-kinase (PI3K)/mammalian target of rapamycin (mTOR) signaling pathway in NSCLC cells. Moreover, the activation of PI3K/mTOR signaling showed an inhibitory effect on the autophagy and radioresistance induced by STMN1 in NSCLC cells. In addition, luciferase reporter assay data indicated that STMN1 was a direct target gene of miR-101, which had been reported to be an inhibitor of autophagy. Based on these data, we suggest that as a target gene of miR-101, STMN1 promotes the radioresistance by induction of autophagy through PI3K/mTOR signaling pathway in NSCLC. Therefore, STMN1 may become a potential therapeutic target for NSCLC radiotherapy.
\end{abstract}

Keywords: radioresistance, non-small-cell lung cancer, STMN1, autophagy, mTOR pathway, PI3K

\section{Introduction}

Lung cancer, the most common invasive cancer, is a leading cause of cancer death worldwide due to its late diagnosis and poor outcomes. ${ }^{1}$ Non-small-cell lung cancer (NSCLC) accounts for approximately $80 \%$ in all cases of lung cancer. ${ }^{1}$ Although novel therapies have been developed, radiation is still an important strategy in NSCLC treatment. However, reduced radiosensitivity of lung cancer cells represents a pivotal obstacle in clinical oncology. Thus, investigation on special therapeutic targets associated with radioresistance shows promises for the enhancement of clinical radiotherapy effect toward NSCLC. ${ }^{2}$

Stathmin1 (STMN1) is a cytosolic phosphoprotein that abundantly expressed in various types of human cancers. STMN1 has been found to destabilize microtubules and play an important role in the regulation of cell cycle progression. ${ }^{3}$ Recently, Nie et $\mathrm{al}^{4}$ found that upregulation of STMN1 correlated with poor differentiation in lung adenocarcinomas, suggesting that deregulation of STMN1 was associated with the development of lung cancer. However, the detailed role of STMN1 in NSCLC, especially in its radioresistance, remains largely unclear. 
Autophagy is a catabolic process that maintains cellular homeostasis. ${ }^{5}$ The role of autophagy in regulating cancer cell apoptosis has been widely investigated, but not fully elucidated. Previous evidence showed that when cancer cells were subjected to unfavorable conditions, such as treatment with anticancer drugs or radiation, autophagy was rapidly activated and functioned as a cytoprotective mechanism to enhance cancer cell survival. ${ }^{6}$ Therefore, autophagy may contribute to the resistance to anticancer therapies including radiation. In addition, microRNAs (miRNAs), a class of short noncoding RNA, have been demonstrated to play a critical role in various biological processes including autophagy and radioresistance by repressing the expression of their target genes at the posttranscriptional level. ${ }^{7}$ For instance, miR-101 has been reported to act as a potent inhibitor of basal, etoposide- and rapamycin-induced autophagy, sensitizing breast cancer cells to 4-hydroxytamoxifen (4-OHT)-mediated cell death by targeting STMN1. ${ }^{8}$ Thus, STMN1 is suggested to be capable of sensitizing tumor cells to chemotherapy by inhibition of autophagy. However, no previous study has investigated the role of STMN1 in radioresistance of NSCLC.

Therefore, the present study mainly aimed to investigate the exact role of STMN1 in the regulation of radioresistance in NSCLC. Besides, we also studied the underlying mechanism involving autophagy and the related signaling pathway.

\section{Materials and methods Cell culture}

NSCLC cell lines, H1299, H292, A549, and H358, and human bronchial epithelial cells (HBE) were obtained from ATCC (American Type Culture Collection, Manassas, VA, USA). H1299, H292, A549, and H358 were cultured in ATCC-formulated F-12K medium supplemented with $10 \%$ fetal bovine serum. HBE cells were cultured in keratinocyteserum free medium with $5 \mathrm{ng} / \mathrm{mL}$ human recombinant epidermal growth factor and $0.05 \mathrm{mg} / \mathrm{mL}$ bovine pituitary extract and supplemented with $0.005 \mathrm{mg} / \mathrm{mL}$ insulin and $500 \mathrm{ng} /$ $\mathrm{mL}$ hydrocortisone. All the cells were cultured at $37^{\circ} \mathrm{C}$ in a humidified incubator containing $5 \% \mathrm{CO}_{2}$.

The Institutional Review Board of Third Xiangya Hospital, Central South University, approved this study and certified that the ethical principles and the design and methods of the research were in accordance with the regulation and procedure requirements.

\section{Cell treatment}

The recombinant lentivirus of Lv-STMN1 (POSL341065641, GENECHEM, Shanghai, People's Republic of China) and anti-STMN1 (PIEL112065641, GENECHEM) were transfected into A549 and H1299 cells, respectively. Then, STMN1 gain model (Lv-STMN1), and STMN1 loss model (anti-STMN1) and the control of gain models (Lv-NC) and the control of loss models (anti-NC) were successfully constructed by selection with G418 (10131-027, Life Technologies, Grand Island, NY, USA). Then, cells in each group were exposed to X-ray irradiation at 2 Gy before a serious analysis.

The STMN1 gain/loss models of A549 and H1299 cells were irradiated by Primus Linear Accelerator (Siemens, Berlin, Germany) at different doses ranging from 0 to $8 \mathrm{~Gy}$ before the clonogenic cell survival assay.

The Lv-STMN1 and Lv-NC cells of A549 and H1299 were treated with or without $5 \mu \mathrm{M}$ of insulin-like growth factor-1 (IGF-1) (BiYunTian, Beijing, People's Republic of China), irradiated by Primus Linear Accelerator (Siemens) at different doses ranging from 0 to $8 \mathrm{~Gy}$ before the clonogenic cell survival assay.

MiR-101 was upregulated or downregulated by transfection with miR-101 mimics or inhibitor, respectively. Pre- and anti-con groups were settled as controls. All the plasmids were purchased from GENECHEM.

\section{Real-time polymerase chain reaction assay}

Total RNA was prepared using a Trizol agent (Life Technologies) according to the manufacturer's instruction. For the analysis of miRNA expression, miScript Reverse Transcription Kit (Qiagen GMbH, Hilden, Germany) was used to convert RNA into cDNA, according to the manufacture's protocol. MiRNA Q-PCR Detection Kit (Qiagen) was used to perform real-time polymerase chain reaction (PCR) on ABI 7500 thermocycler (Invitrogen Life Technologies, Grand Island, NY, USA). U6 gene was used as an endogenous control. For the analysis of mRNA expression, RevertAid $^{\text {TM }}$ H Minus First-strand cDNA Synthesis Kit (Thermo Fisher Scientific, Waltham, MA, USA) was used to convert RNA into cDNA, and real-time PCR was then performed by using the Power SYBR Green kit (BioRad, Hercules, CA, USA) on ABI 7500 thermocycler. Beta-actin was used as an endogenous control. The relative expression was analyzed by the $2^{-\Delta \Delta C t}$ method. The primers for miR-101 (HmiRQP0021) and U6 (HmiRQP9003) were designed and purchased from GeneCopoeia (Guangzhou, People's Republic of China). The primers for STMN1 are shown as follows: sense, 5'-TCGGACTGAGCAGGACTTTC-3' and antisense, 5'-ATGGGACTTGCGTCTTTCTT-3'. 
The primers for $\beta$-actin were shown as follows: sense, 5'-AGGGGCCGGACTCGTCATACT-3' and antisense, 5'-GGCGGCACCACCATGTACCCT-3'.

\section{Western blot}

A total $60 \mu \mathrm{g}$ of proteins were separated on $15 \%$ sodium dodecyl sulfate polyacrylamide gel electrophoresis, (PA003D; Auragene, Changsha, People's Republic of China) transferred to polyvinylidene fluoride membranes (Millipore, Bedford, MA, USA) and probed with primary antibodies: anti-LC3B antibody (Abcam, Hong Kong), anti-Beclin1 antibody (Epitomics, Hong Kong), anti-STMN1 antibody (Abcam), antiphosphoinositide 3-kinase (anti-PI3K) antibody (Santa Cruz Biotechnology, Dallas, TX, USA), anti-P-PI3K (Santa Cruz Biotechnology), anti-mammalian target of rapamycin (mTOR) antibody (Abcam), anti-p-mTOR (Abcam), anti-S6K (Abcam), anti-P-S6K antibody (Abcam), or anti- $\beta$-actin antibody (Boster, Wuhan, People's Republic of China) at $4{ }^{\circ} \mathrm{C}$ for one night, and followed by secondary antibodies conjugated with horseradish peroxidase at room temperature for 1 hour. The protein bands were visualized by the Amersham ECL system (RPN998, GE, Fairfield, CN, USA) and scanned. Data was analyzed by densitometry using Image-Pro plus software 6.0 (Media Cybernetics, Rockville, MD, USA) normalized to $\beta$-actin expression.

\section{Clonogenic cell survival assays}

Cells were irradiated in suspension in F-12K medium with 0,2 , 4,6 , and 8 Gy X-ray radiation at a distance of $20 \mathrm{~cm}$ from the source. An appropriate number of cells were plated into each of five $10 \mathrm{~mm}$ dishes containing $10 \mathrm{~mL}$ F-12K medium. Cells were incubated for 14 days, fixed in methanol for 15 minutes, stained with Giemsa (Sigma-Aldrich Co., St Louis, MO, USA) for 10 minutes, dried in air and colonies counted. The number of colonies derived from irradiated cells was expressed as a percentage of colonies in unirradiated control plates.

\section{Flow cytometric analysis of apoptosis with Annexin-V/PI double staining}

Annexin $\mathrm{V}$ apoptosis detection kit (Life technologies, USA) was used for analysis of apoptosis. After indicated treatment, A549 and H1299 cells were trypsinized, collected, and resuspended. Approximately $2 \times 10^{5}$ cells were harvested and washed twice with cold phosphate buffer saline, then resuspended in $500 \mu \mathrm{L}$ binding buffer. A total of $10 \mu \mathrm{L}$ Annexin V-FITC and $10 \mu \mathrm{L}$ propidium iodide were added to the solution and mixed well. After 15 minutes incubation, the cells were analyzed using flow cytometric analysis (BD Biosciences, San Jose, CA, USA).

\section{Dual luciferase report system}

Wild type (wt) and mutant (mut) 3'-UTR of STMN1 were inserted into downstream of the dual luciferase reporter vector. For luciferase assay, $10^{5}$ cells were plated and cultured in 24-well plates to reach approximately $70 \%$ confluence. Cells were co-transfected with miR-101 mimic and $\mathrm{wt} /$ mut 3'-UTR of STMN1 dual luciferase reporter vector, respectively. After 48 hours transfection, dual luciferase reporter gene assay kit (BioVision, Milpitas, CA, USA) was used to determine the luciferase activities on luminometer (Roche, Basel, Switzerland). Renilla luciferase activity was normalized to firefly luciferase activity.

\section{Electron microscopy}

Cell pellets were fixed in $2.3 \%$ glutaraldehyde, postfixed in $2 \%$ osmium tetroxide and $0.5 \%$ uranyl acetate, dehydrated and embedded in Spurs epoxy resin. Ultrathin sections $(90 \mathrm{~nm})$ were made and double-stained with uranyl acetate and lead citrate, and viewed with a Philips CM10 transmission electron microscope (Phillips Electronics, Amsterdam, the Netherlands).

\section{Statistical analysis}

Analysis of data was performed by using Graphpad prism5 software (GraphPad Software, Inc., La Jolla, CA, USA). Student $t$-tests or one-way analysis of variance (ANOVA) was used depending on the experimental conditions. Results showed in figures are expressed as mean \pm standard deviation (SD). Statistical significance was evaluated by $P$-values of $<0.05$.

\section{Results \\ Upregulation of STMNI is associated with the activation of autophagy induced by X-ray in NSCLC cells}

Western blotting assay was first conducted to determine the protein levels of STMN1 in NSCLC cell lines. As shown in Figure 1A, the expression of STMN1 was significantly increased in NSCLC cell lines (H1299, A549, H358, and H292) compared to HBE. Furthermore, A549 and H1299 cells were exposed to X-irradiation (0, 2, and $4 \mathrm{~Gy}$ ). We found that STMN1 was significantly upregulated in A549 and H1299 cells after exposure to X-irradiation (Figure 1B). Meanwhile, the autophagy-related gene Beclin1 and LC3II/I were also significantly increased, indicating that the level of autophagy was increased (Figure 1B). These data suggest that upregulation of STMN1 may be associated with the activation of autophagy induced by $\mathrm{X}$-irradiation. 

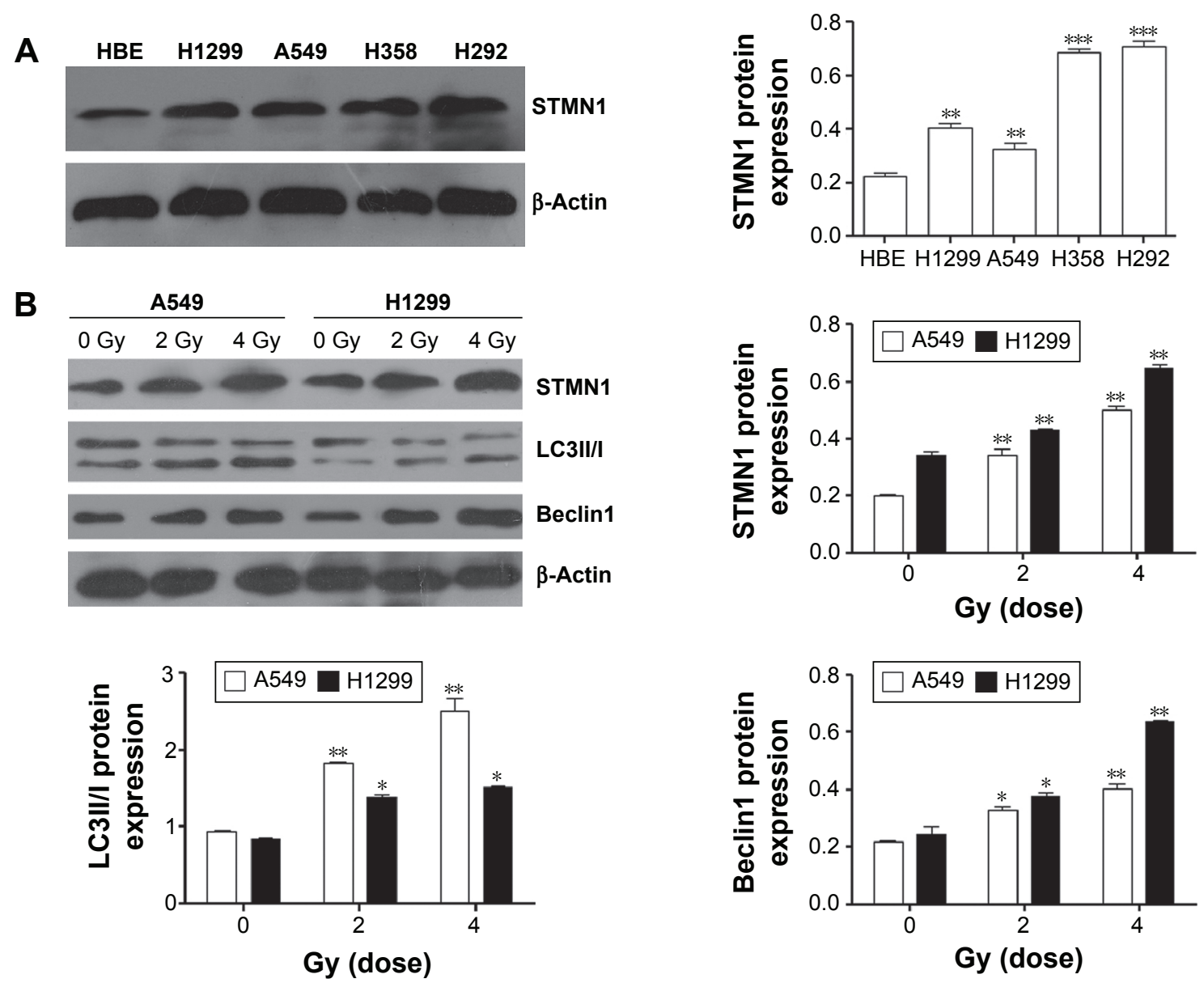

C

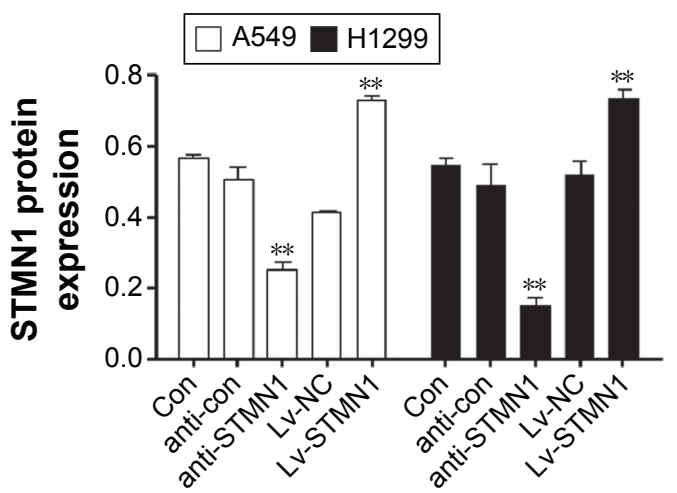

Figure I Upregulation of STMNI is associated with autophagy enhanced by X-irradiation in A549 and HI299 cells.

Notes: (A) STMNI was dramatically upregulated in NSCLC cell lines compared to HBE by Western blot method. (B) The protein expression level of STMNI, BeclinI, and LC3II/I was upregulated when A549 and HI299 cells were treated with X-ray irradiation at 2 and 4 Gy compared with 0 Gy control groups measured with Western blot. (C) The protein expression levels of STMNI in STMNI-up/downregulating of A549 and HI299 cells by Western blot analysis. Data presented as mean \pm SD, $* P<0.05$, $* * P<0.01$, **** $<<0.001$.

Abbreviations: anti-con, the control of anti-STMNI loss models; anti-STMNI, STMNI loss model; con, control; HBE, human bronchial epithelial cells; Lv-NC, the control of gain models; Lv-STMNI, STMNI gain model; NSCLC, non-small-cell lung cancer; STMNI, Stathmin I; SD, standard deviation.

\section{Downregulation of STMNI sensitizes A549 and HI299 cells to radiation}

The upregulation and knockdown of STMN1 cell models were successfully constructed by using A549 and H1299 cells, which were confirmed by Western blot analysis (Figure 1C). To reveal the role of STMN1 in the sensitivity of NSCLC cells to radiotherapy, cells in each group were exposed to X-ray (4 Gy). And 48 hours after radiation, the apoptosis levels in each group were determined by using flow cytometric assay. As shown in Figure 2A, the apoptotic levels were significantly increased after knockdown of STMN1, compared to the control group. On the contrary, upregulation 
A
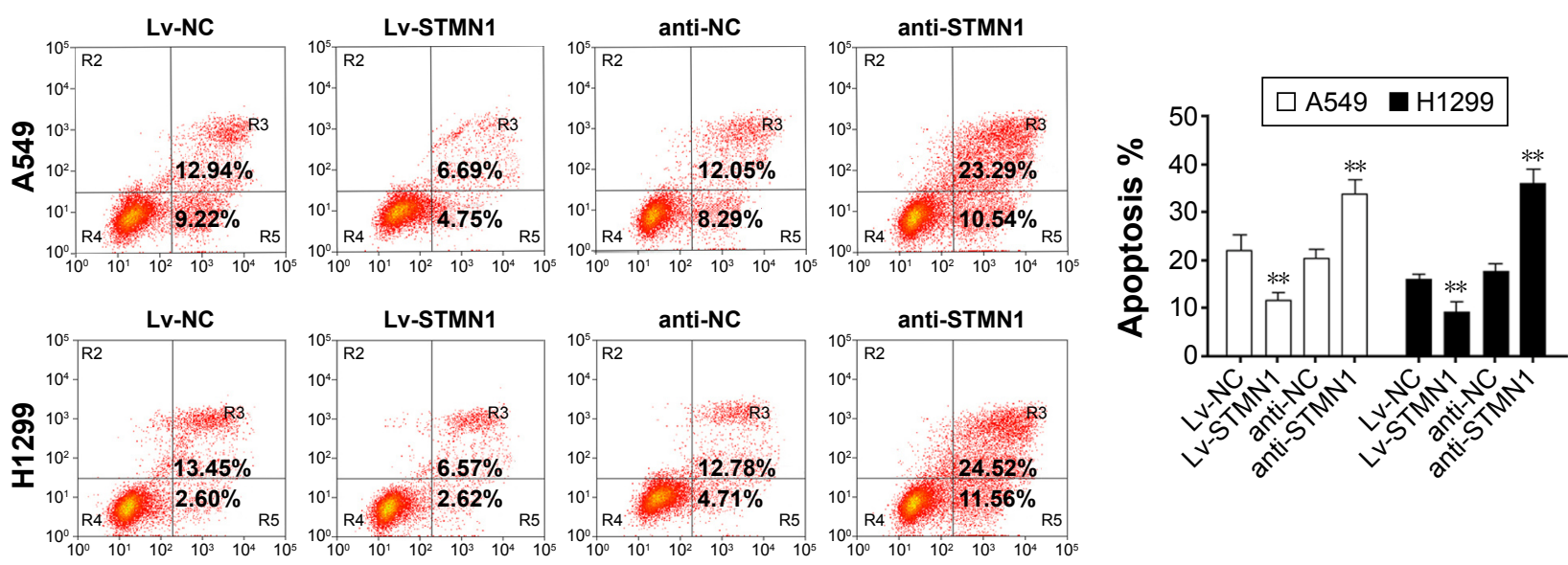

B
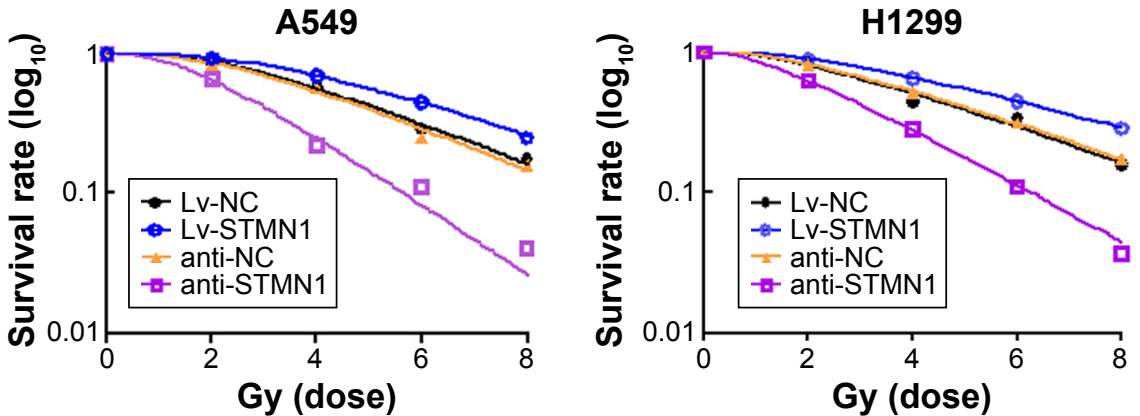

Figure 2 Downregulation of STMNI increased radiation sensitivity of A549 and HI 299 cells.

Notes: (A) FACS analysis of apoptotic in STMNI-up/downregulating of A549 and HI 299 cells, when they were subjected to X-ray (2 Gy). (B) The capacity of cell colony formation in STMNI-up/downregulating of A549 and HI 299 cells, when they were subjected to X-ray $(0,2,4,6$, and $8 \mathrm{~Gy})$. Data presented as mean \pm SD, $* * P<0.0 \mathrm{I}$. Abbreviations: anti-NC, the control of loss models; anti-STMNI, STMNI loss model; FACS, fluorescence-activated cell sorting; Lv-NC, the control of gain models; Lv-STMNI, STMNI gain model; STMNI, Stathmin I; SD, standard deviation.

of STMN1 led to a significant decrease in apoptotic levels in NSCLC cells, compared to the control group (Figure 2A). These findings suggest that inhibition of STMN1 expression sensitizes NSCLC cells to radiation.

To further validate the role of STMN1 silencing in the radiosensitivity of NSCLC cells, cells in each group were subjected to X-ray $(0,2,4,6$, and 8 Gy). Then, the colony formation assay was conducted to test cell survival. Our data showed that the surviving rate of anti-STMN1 groups was significantly decreased compared with that of anti-NC cells and the control cells groups (Figure 2B). On the contrary, the surviving rate of Lv-STMN1 cells groups was markedly increased compared with that of Lv-NC cells and control cells groups (Figure 2B). These results suggest that inhibition of STMN1 expression indeed enhances the sensitivity of A549 and H1299 cells to X-irradiation.

\section{Knockdown of STMNI inhibited radiation-induced autophagy in NSCLC cells}

We further tested the effect of STMN1 knockdown or overexpression on X-irradiation-induced autophagy in NSCLC cells. Western bolt analysis indicated that the expression of Belclin1 and the ratio of LC-3II to LC-3I were increased in the LV-STMN1 group but reduced in the anti-STMN1 group, when compared to the control groups (Figure 3A). After that, the autophagosomes in each group were characterized by electronic microphotography. Images of electron microscopy showed the numbers and the volumes of autophagosomes were significantly increased in Lv-STMN1 group, but dramatically decreased in anti-STMN1 group (Figure 3B), indicated that the autophagy level was reduced after knockdown of STMN1 in NSCLC cells. Based on these data, we suggest that knockdown of STMN1 significantly inhibits the radiation-induced autophagy in NSCLC cells.

\section{STMNI enhanced the radiation-induced autophagy and radioresistance through inhibition of $\mathrm{PI} 3 \mathrm{~K} / \mathrm{mTOR}$ signaling in NSCLC cells}

As P13K/mTOR signaling pathway has been demonstrated to be involved in autophagy in human cancers, we further examined the activity of the $\mathrm{P} 13 \mathrm{~K} / \mathrm{mTOR}$ signaling pathway by performing Western blot analysis. As shown in 
A
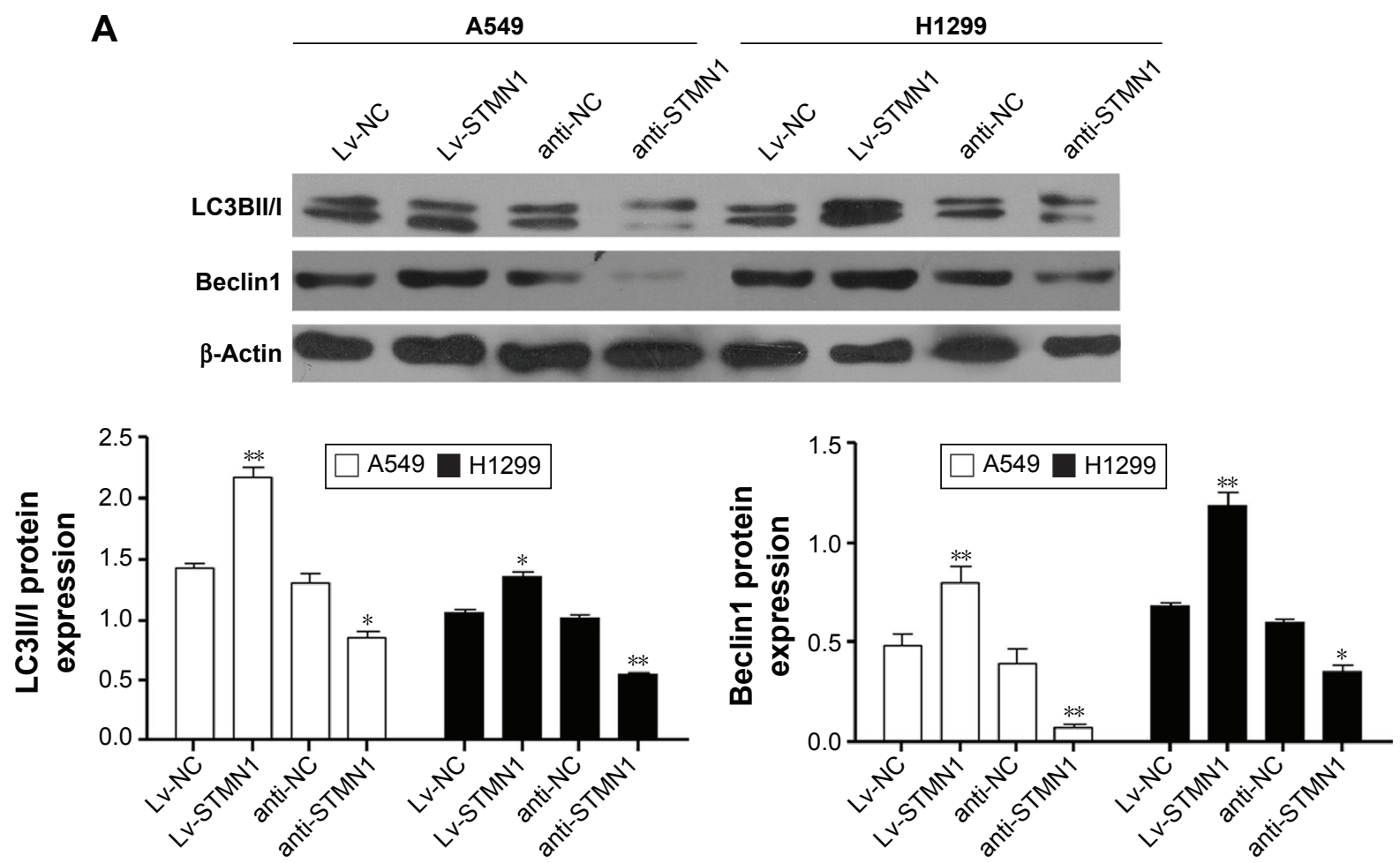

B
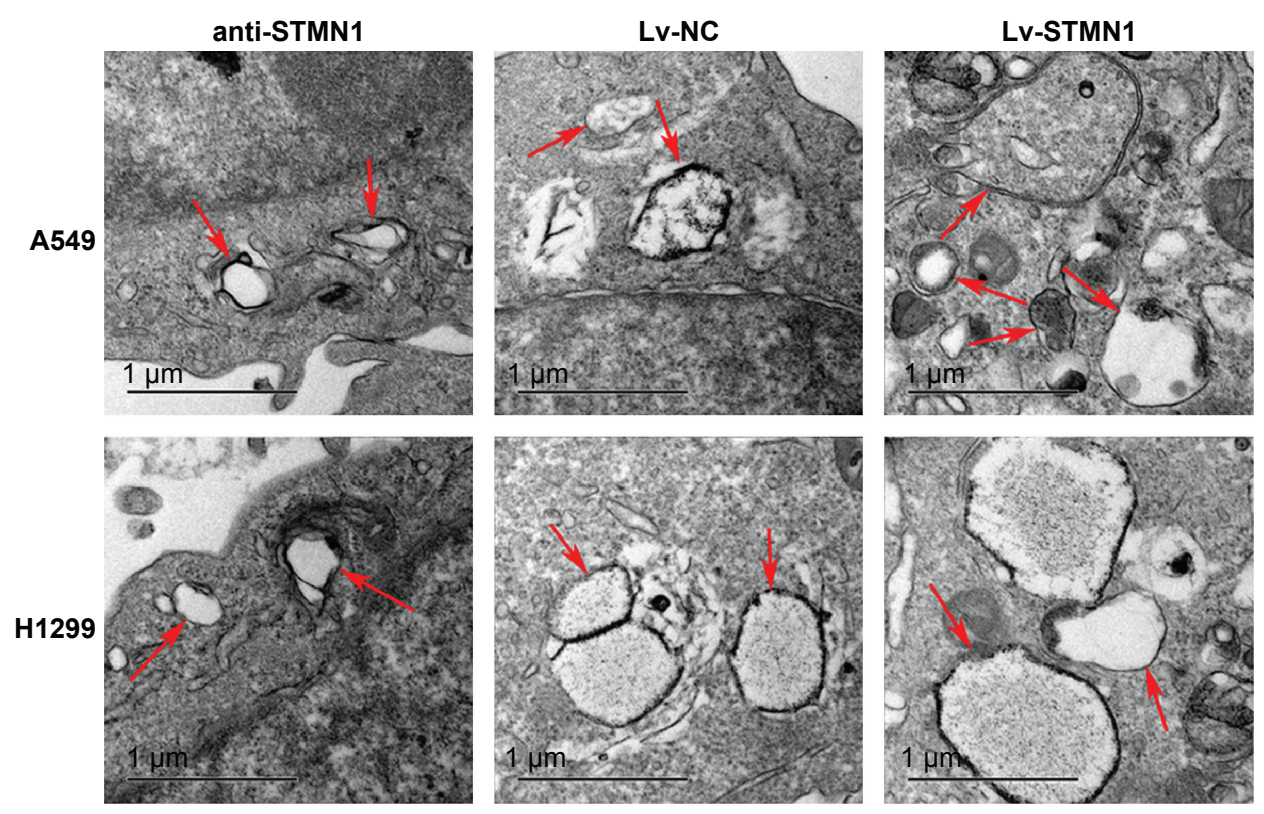

Figure 3 STMNI regulated autophagy in A549 and HI299 cells.

Notes: (A) The protein expression level of Beclin I and LC3II/I in STMNI-up/downregulating of A549 and HI299 cells, which were treated with X-ray irradiation at 2 Gy. (B) Autophagosome-like structures (indicated by the red arrows) were assayed by TEM. Data presented as mean $\pm S D, * P<0.05$, $* * P<0.01$.

Abbreviations: anti-NC, the control of loss models; anti-STMNI, STMNI loss model; Lv-NC, the control of gain models; Lv-STMNI, STMNI gain model; STMNI, Stathmin I; SD, standard deviation; TEM, transmission electron microscopy.

Figure 4A, overexpression of STMN1 led to a significant decrease in the levels of p-P13K, p-mTOR, and p-S6K, when compared with those in the Lv-NC cells, indicating that the activity of PI3K/mTOR signaling was downregulated. On the contrary, knockdown of STMN1 caused a significant increase in the levels of p-P13K, p-mTOR, and p-S6K, when compared with those in the anti-NC cells, indicating that the activity of PI3K/mTOR signaling was upregulated (Figure 4A). These data suggest that knockdown of STMN1 may activate the PI3K/mTOR signaling, which further inhibit the radiation-induced autophagy in NSCLC cells exposed to radiation. 
A

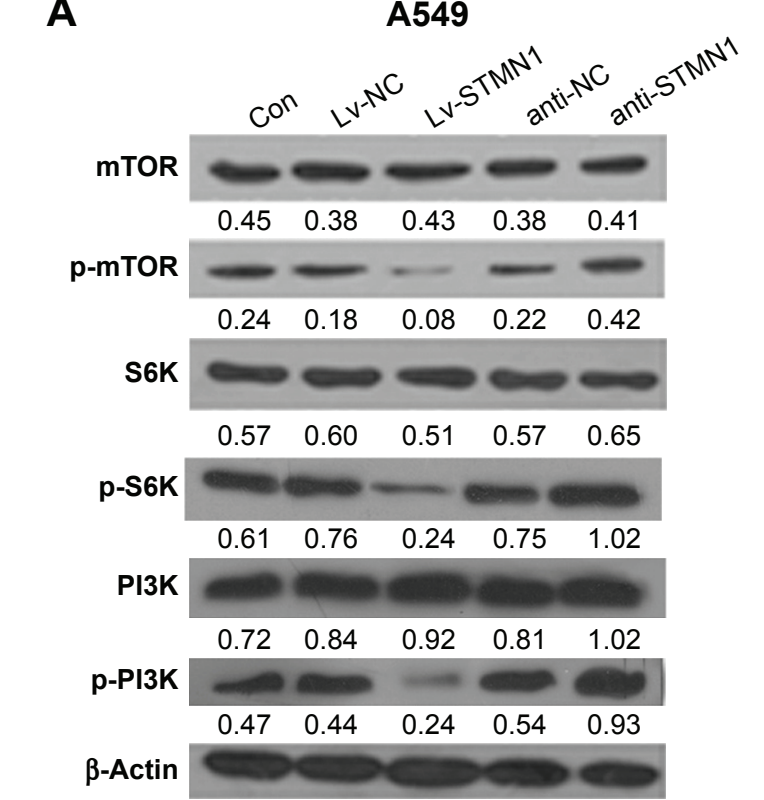

H1299

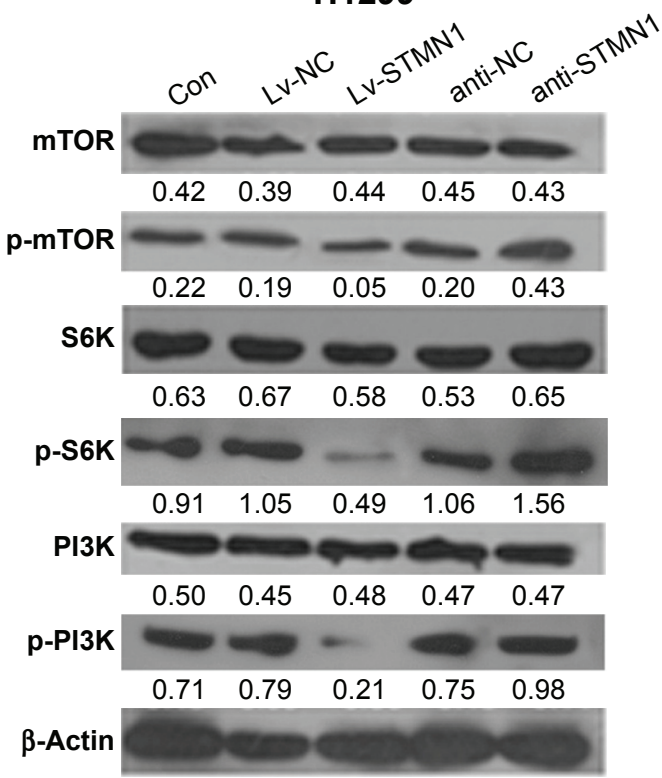

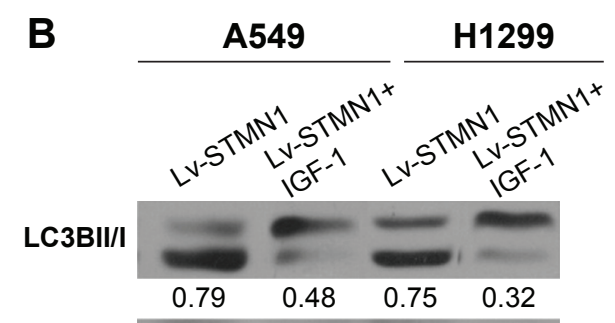

Beclin1
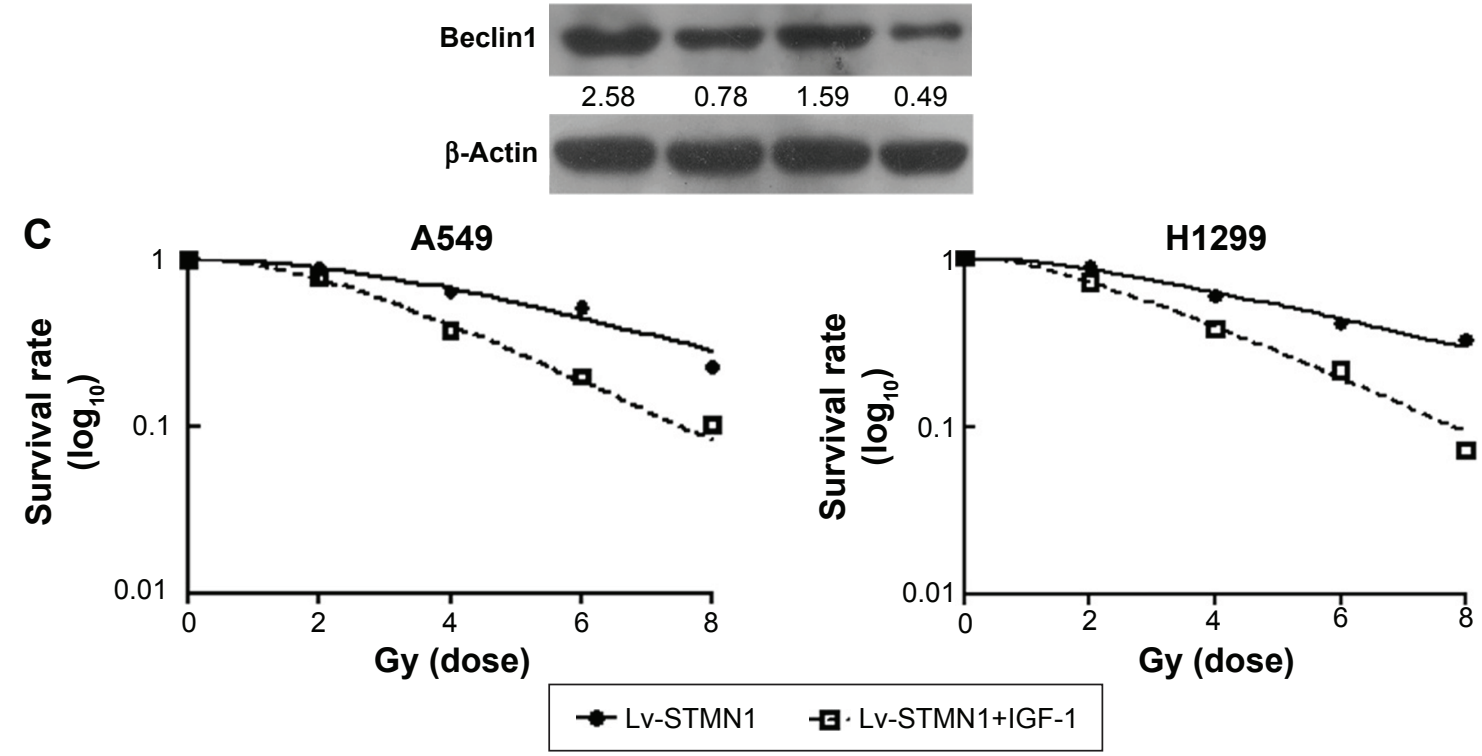

Figure 4 STMNI regulated the autophagy ability through PI3K/mTOR pathway in A549 and HI299 cells.

Notes: (A) Western blot analyzed the protein expression levels of mTOR and PI3K signal pathway in STMNI-up/downregulating of A549 and HI299 cells, which were treated with X-ray at 2 Gy. (B) Western blot analysis of LC3-I and LC3-II, BeclinI expression in STMNI-overexpressing A549 and HI299 cells treated with or without IGF-I, when they subjected to X-ray in 2 Gy. (C) And the clonogenic cell survival assay was conducted. Data presented as mean \pm SD.

Abbreviations: con, control; anti-NC, the control of loss models; anti-STMNI, STMNI loss model; IGF-I, insulin-like growth factor-I; Lv-NC, the control of gain models; Lv-STMNI, STMNI gain model; mTOR, mammalian target of rapamycin, p, phosphorylation; STMNI, Stathmin I; SD, standard deviation.

To further confirm these findings, STMN1-overexpressing A549 and H1299 cells were treated with the PI3K/mTOR signaling agonist IGF-1, followed by X-ray treatment. As shown in Figure 4B, Lv-STMN1+IGF-1 group showed a significant decrease in the protein level of Beclin1 and the ratio of LC3II to LC3I, compared to the control groups, indicating that the autophagy level is decreased. Therefore, activation of PI3K/mTOR signaling suppressed the promoting effect 
of STMN1 overexpression on autophagy in NSCLC cells exposed to X-ray. We further determined the radiosensitivity in each group. As shown in Figure 4C, the surviving rate of NSCLC cells in the Lv-STMN1+IGF-1 group was significantly decreased compared to the control group, indicating that activation of PI3K/mTOR signaling suppressed the STMN1 overexpression-induced radioresistance in NSCLC cells exposed to X-ray. Based on these data, we demonstrated that STMN1 enhanced the radiation-induced autophagy and radioresistance through inhibition of $\mathrm{P} 13 \mathrm{~K} / \mathrm{mTOR}$ signaling in NSCLC cells.

\section{STMNI is a direct target of miR-I0I in NSCLC cells}

We further used web-based application of bioinformatic tools to analyze whether STMN1 is mediated miRs, and our data showed that miR-101 may potentially target to the 3'UTR of the STMN1 mRNA (Figure 5A). To clarify this predication, we cloned the 3'UTR of STMN1 downstream to a luciferase reporter gene, and its mutant version was also constructed (Figure 5A). The constructed vectors were co-transfected with miR-101 mimics or inhibitor into A549 and H1299 cells. The luciferase activity of cells transfected with miR-101 and

A

\begin{tabular}{|c|c|c|}
\hline & Firefly luciferase & STMN1-3'UTR \\
\hline \multicolumn{3}{|c|}{ Position 270-292 of STMN13'UTR } \\
\hline & $\begin{array}{c}5^{\prime} \quad \operatorname{cug} G \in \cup \cup A \\
: \quad|\quad|\end{array}$ & $\begin{array}{c}A \cup G G C \cup A G \cup A C \cup G \cup a \\
\end{array}$ \\
\hline hsa-miR-101 & $\begin{aligned} 3^{\prime} \text { aag } \cup C A A \\
:|||l|\end{aligned}$ & 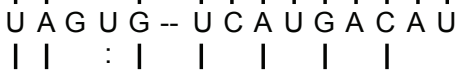 \\
\hline MN1-Mut3'UTR & $5^{\prime} \quad \operatorname{cug} G \mathrm{G} \cup \cup \mathrm{A}$ & $A \cup G G C \cup A C \cup \cup C A G A a$ \\
\hline
\end{tabular}

B

A549

$\square$ STMN1-3'UTR $\square$ Mut-STMN1-3'UTR

H1299

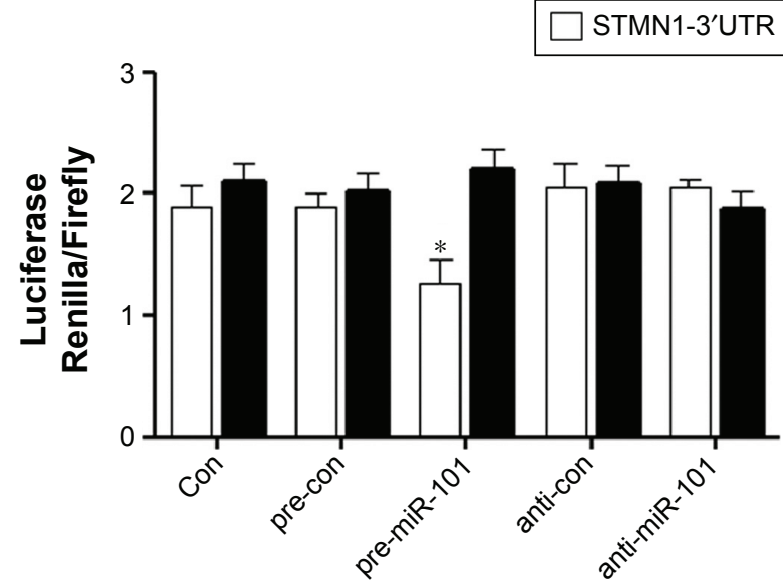

C

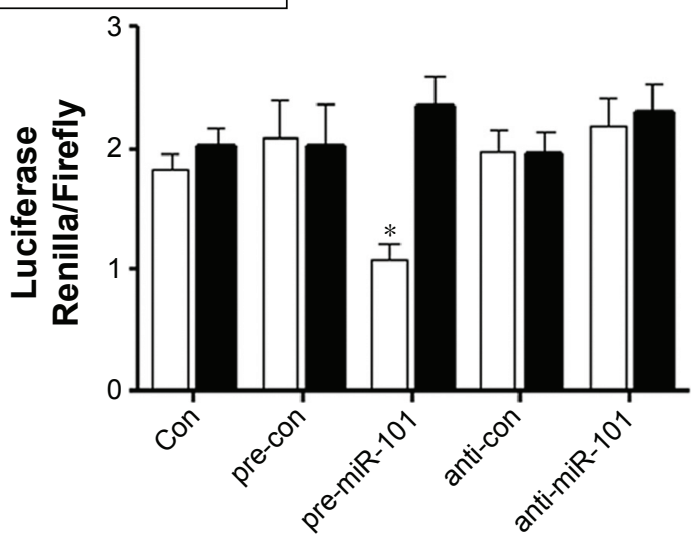

A549

H1299

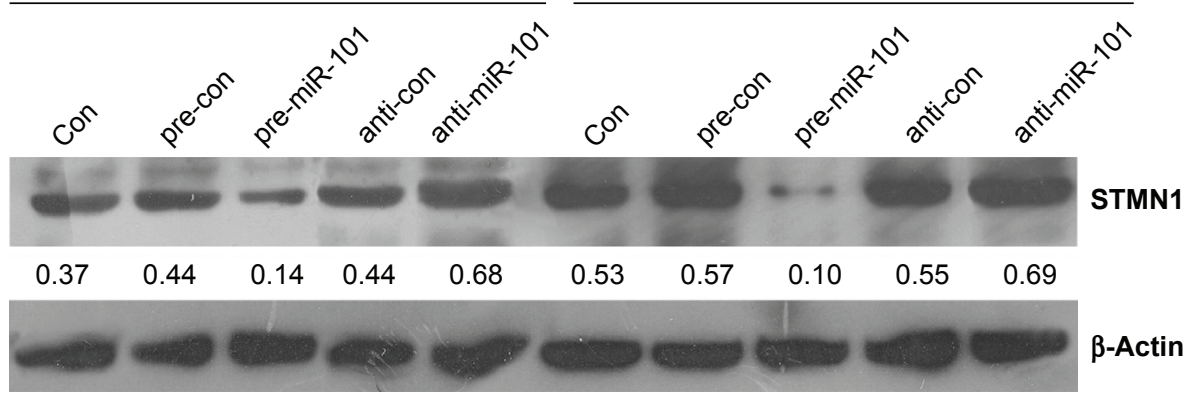

Figure 5 STMNI was a target gene of miR-I0I.

Notes: (A) The binding sites of hsa-miR-I0I of STMNI mRNA 3'UTR and mutation of STMNI mRNA 3'UTR. (B) The repression of luciferase activity by STMNI 3'UTR was dependent on miR-I0I. Mutated STMNI 3'UTR abrogated miR-I0I mediated repression luciferase activity in A549 and HI299 cells. (C) Western blot analyzed the protein expression levels of STMNI after transfecting with miR-I0I mimic, miR-I0I inhibitor, and negative controls in A549 and HI299 cells. Data presented as mean \pm SD. $* P<0.05$.

Abbreviations: anti-con, the control of anti-STMNI loss models; con, control; hsa, homo sapien; STMNI, Stathmin I; SD, standard deviation. 
STMN1 was significantly decreased compared to inhibitor control groups (Figure 5B). However, the miR-101-mediated repression of luciferase activity was abolished by the mutant putative binding site (Figure 5B). In addition, Western blotting analysis data also confirmed that miR-101 negatively regulated the protein expression of STMN1 in NSCLC A549 and H1299 cells (Figure 5C). According to the earlier data, STMN1 is indeed a target gene of miR-101 in NSCLC cells.

\section{Discussion}

Our study aimed to reveal the role of STMN1 in radioresistance in NSCLC as well as the underlying mechanism. We first found that STMN1 was significantly upregulated in NSCLC cell lines. Moreover, the radioresistant NSCLC cells showed an upregulation of STMN1. Meanwhile, the autophagy-related Beclin1 level as well as the ratio of LC3BII to LC3BI was also significantly increased with the increasing X-ray dose in NSCLC cells. It has been well established that high levels of autophagic Beclin1 and LC3BII/I are involved in the chemoresistance and radioresistance,, 10 and the tumor-promoting role of autophagy has been reported in the mouse model of lung cancer. ${ }^{11}$ Thus, we suggest that STMN1 is associated with the radiosensitivity in lung cancer cells.

In the present study, we found that the enhancement of autophagy was along with an increase of STMN1 expression in radioresistant NSCLC cells. By gain- and lossfunction experiments, we found that downregulation of STMN1 resulted in a decrease of autophagy, subsequently sensitized NSCLC A549 and H1299 cells to radiation by inducing proliferation inhibition and promoting apoptosis. STMN1 has been found to be associated with chemoresistance, including esophageal squamous cell cancer, ${ }^{12}$ breast cancer, ${ }^{13}$ colorectal cancer, ${ }^{14}$ and lung cancer. ${ }^{4}$ Silencing of STMN1 combined with paclitaxel significantly inhibited the proliferation of esophageal squamous cell cancer cells, with a significantly higher apoptosis rate. ${ }^{12} \mathrm{Wu}$ et $\mathrm{al}^{14}$ found that silencing STMN1 also significantly improved chemoresponse to colorectal cancer therapeutic agent 5-fluorouracil treatment. Moreover, the expression of STMN1 was higher in NSCLC tissues compared to normal tissues, and a high level of STMN1 was often correlated with poor prognosis. The STMN1 was higher in taxol-resistant NSCLC NCI-H1299 cells than in NCI-H1299 cells. ${ }^{13}$ Inhibition of STMN1 expression increased the sensitivity to taxol and promoted cellular apoptosis in taxol-resistant NSCLC cells. ${ }^{13}$ The evidence from our research data also demonstrated the silencing of STMN1 which sensitizes NSCLC cells to radiation.
It was reported that high-phosphorylated Stathmin was associated with several PI3K/mTOR pathway alterations, including increased PIK3CA copy number and PI3K activation, ${ }^{15}$ indicating that $\mathrm{STMN1}$ may respond to PI3K/mTOR inhibitors. Schimmack et $\mathrm{al}^{16}$ found that STMN1 knockdown decreased proliferation, and PI3K inhibitors directly inhibited proliferation via Stathmin inactivation. Here we found that overexpression of STMN1 in NSCLC cells decreased PI3K/mTOR signaling and increased autophagy, resulting in decreased apoptosis and resistance to radiation, which was rescued by $\mathrm{PI} 3 \mathrm{~K} / \mathrm{mTOR}$ signaling agonist IGF-1. Thus, we provided evidence to illustrate that inhibition of STMN1 sensitized NSCLC cells to radiation via regulating PI3K/mTOR signaling and autophagy. Previous study also showed that Stathmin was involved in the hypoxiamediated PI3K/serine/threonine kinase AKT/mTOR pathway activation in ovarian clear cell adenocarcinoma. ${ }^{17}$ Besides, STMN1 is a relevant $\mathrm{p} 27$ binding partner, and their cooperation controls the early phase of G1 to S phase transition. ${ }^{18}$ Upregulation of STMN1 has been found to cause a loss of cell cycle mitotic checkpoint control, and thus may render tumors amenable to PI3K inhibitory therapy. ${ }^{16}$

In addition, STMN1 has been identified as a target of miR-101, regulating STMN1 expression at transcriptional and translational levels in other cancer types. ${ }^{19}$ In the present study, we also confirmed that STMN1 was a direct target gene of miR-101 in NSCLC cells. It was reported that downregulation of miR-101 was associated with a poor prognosis in patients with NSCLC. ${ }^{20}$ Chen et $\mathrm{al}^{21}$ demonstrated that the endogenous miR-101 level was lower in 13 radioresistant NSCLC cell lines. Enforced expression of miR-101 could inhibit NSCLC cell proliferation and invasion, and sensitize NSCLC cells to paclitaxel-mediated apoptosis..22 Besides, miR-101 also sensitized the A549 NSCLC cell line, ${ }^{23}$ epithelial ovarian cancer cells, and human bladder cancer cells to cisplatin via the activation of caspase 3-dependent apoptosis. $^{24,25} \mathrm{Xu}$ et $\mathrm{al}^{26}$ recently reported that miR-101 repressed hepatocellular cancer progression through directly targeting EZH2 oncogene and sensitized liver cancer cells to doxorubicin or fluorouracil to induce apoptosis by inhibiting autophagy. Thus, the miR-101-mediated sensitivity to radiation as well as autophagy may via directly targeting STMN1 in NSCLC cells.

In conclusion, we find that STMN1, a target of miR-101, can regulate autophagy and sensitivity to radiation through $\mathrm{PI} 3 \mathrm{~K} / \mathrm{mTOR}$ signaling in NSCLC. Our findings suggest that silencing of STMN1 or upregulation of miR-101 could be a radiotherapy enhancer in the lung cancer cell lines, 
which may play a synergistic effect with PI3K inhibitory therapy.

\section{Acknowledgment}

This work was supported by the National Natural Science Foundation of China (studies on the molecular mechanism of E1A enhanced the sensitivity of cervical carcinoma in radiotherapy by E6/E6AP; number 81372792).

\section{Disclosure}

The authors report no conflicts of interest in this work.

\section{References}

1. Ueki N, Matsuo Y, Togashi Y, et al. Impact of Pretreatment interstitial lung disease on radiation pneumonitis and survival after stereotactic body radiation therapy for lung cancer. $J$ Thorac Oncol. 2015;10(1): $116-125$.

2. Cherni I, Weiss GJ. miRNAs in lung cancer: large roles for small players. Future Oncol. 2011;7(9):1045-1055.

3. Singer S, Malz M, Herpel E, et al. Coordinated expression of stathmin family members by far upstream sequence element-binding protein-1 increases motility in non-small cell lung cancer. Cancer Res. 2009;69(6): 2234-2243.

4. Nie W, Xu MD, Gan L, Huang H, Xiu Q, Li B. Overexpression of stathmin 1 is a poor prognostic biomarker in non-small cell lung cancer. Lab Invest. 2015;95(1):56-64.

5. Del BD, Desideri M, De Luca T, et al. Histone deacetylase inhibition synergistically enhances pemetrexed cytotoxicity through induction of apoptosis and autophagy in non-small cell lung cancer. Mol Cancer. 2014; 13:230.

6. Peng PL, Kuo WH, Tseng HC, Chou FP. Synergistic tumor-killing effect of radiation and berberine combined treatment in lung cancer: the contribution of autophagic cell death. Int J Radiat Oncol Biol Phys. 2008; 70(2):529-542.

7. Booton R, Lindsay MA. Emerging role of MicroRNAs and long noncoding RNAs in respiratory disease. Chest. 2014;146(1):193-204.

8. Frankel LB, Wen J, Lees M, et al. microRNA-101 is a potent inhibitor of autophagy. EMBO J. 2011;30(22):4628-4641.

9. Chatterjee A, Chattopadhyay D, Chakrabarti G. miR-17-5p downregulation contributes to paclitaxel resistance of lung cancer cells through altering beclin1 expression. PLoS One. 2014;9(4):e95716.

10. Wang W, Fan H, Zhou Y, Duan P, Zhao G, Wu G. Knockdown of autophagy-related gene BECLIN1 promotes cell growth and inhibits apoptosis in the A549 human lung cancer cell line. Mol Med Rep. 2013;7(5): $1501-1505$.

11. Chang SH, Minai-Tehrani A, Shin JY, et al. Beclin1-induced autophagy abrogates radioresistance of lung cancer cells by suppressing osteopontin. J Radiat Res. 2012;53(3):422-432.
12. Feng W, Xiaoyan X, Xuan Y, et al. Silencing stathmin-modulating efficiency of chemotherapy for esophageal squamous cell cancer with paclitaxel. Cancer Gene Ther. 2015;22(3):115-121.

13. Lin X, Liao Y, Xie J, Liu S, Su L, Zou H. Op18/stathmin is involved in the resistance of taxol among different epithelial carcinoma cell lines. Cancer Biother Radiopharm. 2014;29(9):376-386.

14. Wu W, Tan XF, Tan HT, Lim TK, Chung MC. Unbiased proteomic and transcript analyses reveal that stathmin-1 silencing inhibits colorectal cancer metastasis and sensitizes to 5-fluorouracil treatment. Mol Cancer Res. 2014;12(12):1717-1728.

15. Wik E, Birkeland E, Trovik J, et al. High phospho-Stathmin(Serine38) expression identifies aggressive endometrial cancer and suggests an association with PI3K inhibition. Clin Cancer Res. 2013;19(9): 2331-2341.

16. Schimmack S, Taylor A, Lawrence B, et al. Stathmin in pancreatic neuroendocrine neoplasms: a marker of proliferation and PI3K signaling. Tumour Biol. 2015;36(1):399-408.

17. Tamura K, Yoshie M, Miyajima E, Kano M, Tachikawa E. Stathmin regulates hypoxia-inducible factor-1alpha expression through the mammalian target of rapamycin pathway in ovarian clear cell adenocarcinoma. ISRN Pharmacol. 2013;2013:279593.

18. Berton S, Pellizzari I, Fabris L, et al. Genetic characterization of p27(kip1) and stathmin in controlling cell proliferation in vivo. Cell Cycle. 2014;13(19):3100-3111.

19. Sun Q, Liu T, Zhang T, et al. miR-101 sensitizes human nasopharyngeal carcinoma cells to radiation by targeting stathmin 1. Mol Med Rep. 2015; 11(5):3330-3336.

20. Luo L, Zhang T, Liu H, et al. MiR-101 and Mcl-1 in non-small-cell lung cancer: expression profile and clinical significance. Med Oncol. 2012; 29(3):1681-1686.

21. Chen S, Wang H, Ng WL, Curran WJ, Wang Y. Radiosensitizing effects of ectopic miR-101 on non-small-cell lung cancer cells depend on the endogenous miR-101 level. Int J Radiat Oncol Biol Phys. 2011;81(5): $1524-1529$.

22. Zhang JG, Guo JF, Liu DL, Liu Q, Wang JJ. MicroRNA-101 exerts tumor-suppressive functions in non-small cell lung cancer through directly targeting enhancer of zeste homolog 2.J Thorac Oncol. 2011; 6(4):671-678

23. Yin J, Wang M, Jin C, Qi Q. miR-101 sensitizes A549 NSCLC cell line to CDDP by activating caspase 3-dependent apoptosis. Oncol Lett. 2014;7(2):461-465.

24. Liu L, Guo J, Yu L, et al. miR-101 regulates expression of EZH2 and contributes to progression of and cisplatin resistance in epithelial ovarian cancer. Tumour Biol. 2014;35(12):12619-12626.

25. Bu Q, Fang Y, Cao Y, Chen Q, Liu Y. Enforced expression of miR101 enhances cisplatin sensitivity in human bladder cancer cells by modulating the cyclooxygenase-2 pathway. Mol Med Rep. 2014;10(4): 2203-2209.

26. Xu L, Beckebaum S, Iacob S, et al. MicroRNA-101 inhibits human hepatocellular carcinoma progression through EZH2 downregulation and increased cytostatic drug sensitivity. J Hepatol. 2014;60(3):590-598.
OncoTargets and Therapy

\section{Publish your work in this journal}

OncoTargets and Therapy is an international, peer-reviewed, open access journal focusing on the pathological basis of all cancers, potential targets for therapy and treatment protocols employed to improve the management of cancer patients. The journal also focuses on the impact of management programs and new therapeutic agents and protocols on

\section{Dovepress}

patient perspectives such as quality of life, adherence and satisfaction. The manuscript management system is completely online and includes a very quick and fair peer-review system, which is all easy to use. Visit http://www.dovepress.com/testimonials.php to read real quotes from published authors. 